\title{
Pacific
}

Journal of

Mathematics

\section{NONLOCAL UNIFORM ALGEBRAS ON THREE-MANIFOLDS}

ALEXANDER J. IZZO

Volume 259 No. 1 


\title{
NONLOCAL UNIFORM ALGEBRAS ON THREE-MANIFOLDS
}

\author{
ALEXANDER J. IZZO
}

\begin{abstract}
The existence of nonlocal uniform algebras was first proven by Eva Kallin in 1963. Here we prove that on every compact $C^{\infty}$-manifold of dimension greater than or equal to three, there exists a nonlocal uniform algebra generated by $C^{\infty}$-smooth functions.
\end{abstract}

Dedicated to the memory of Walter Rudin

\section{Introduction}

Let $A$ be a uniform algebra on a compact Hausdorff space $X$. A function $f$ in $C(X)$ is said to belong locally on $X$ to $A$ if for each point $x$ in $X$, there are a neighborhood $N$ of $x$ and a function $g$ in $A$ coinciding with $f$ on $N$. The algebra $A$ is said to be local on $X$ if every function that belongs locally on $X$ to $A$ is in $A$. The algebra $A$ is said to be local if it is local on its maximal ideal space $\mathfrak{M}_{A}$. It was conjectured for some time that every uniform algebra is local in this sense. This conjecture was disproved by Kallin [1963], who gave an example of a compact set $X$ in $\mathbb{C}^{4}$ such that $P(X)$ (the uniform closure on $X$ of the polynomials in $\left.z_{1}, \ldots, z_{4}\right)$ is nonlocal.

In [Izzo 2010], we studied localization for uniform algebras generated by smooth functions on two-manifolds. The results there suggest that perhaps these uniform algebras are always local. However, it is also shown there that not every uniform algebra generated by smooth functions on a manifold is local. Specifically, on every compact manifold of dimension greater than or equal to four, there exists a nonlocal uniform algebra generated by smooth functions. The question thus arises whether there exist nonlocal uniform algebras generated by smooth functions on manifolds of lower dimension. Here we show that such algebras exist on every three-manifold. No such uniform algebras exist on one-manifolds because if $J$ is a compact one-manifold (possibly with boundary) and $A$ is a uniform algebra on $J$ generated by smooth functions and with maximal ideal space $J$, then $A=C(J)$. (Proof: By the Stone-Weierstrass theorem, it suffices to show the real-valued functions in $A$ separate points. Given $p \neq q$ in $J$, choose a smooth function $f$ in $A$ whose real part separates $p$ from $q$. Then $f(J)$ has two-dimensional Lebesgue measure zero in $\mathbb{C}$. Hence, by the Hartogs-Rosenthal theorem [Gamelin 1984,

MSC2010: 46J10, 46J15, 30H50, 30J15.

Keywords: nonlocal uniform algebra, three-manifold. 
II.8.4], the real coordinate function $x$ is a uniform limit on $f(J)$ of rational functions with poles off $f(J)$. Because $\mathfrak{M}_{A}=J$, it follows that the real part of $f$ is in $A$.) The question whether a uniform algebra generated by smooth functions and whose maximal ideal space is a two-manifold must be local remains open.

The space on which Kallin's nonlocal uniform algebra is defined cannot be embedded in a three-manifold, so the approach used to construct nonlocal uniform algebras on four-manifolds in [Izzo 2010] does not work for three-manifolds. Instead we use an approach to nonlocal uniform algebras due to Sidney [1968]. Our proof is also related to the Beurling-Rudin theorem on the closed ideals in the disc algebra [Rudin 1957] (or see [Hoffman 1962, pp. 82-89]).

It is with a mixture of joy and sorrow that I dedicate this paper to the memory of Walter Rudin. Sorrow, of course, that he is no longer with us; joy that, through his work and his influence on others, he will in some sense always be with us. The breadth of Rudin's research contributions has been a great inspiration to me and many other mathematicians. In addition, Rudin had a profound impact on my mathematical development and view of the subject. My first exposure to analysis was with his Principles of mathematical analysis [Rudin 1976]; I learned real and complex analysis from his Real and complex analysis [Rudin 1974] and functional analysis from his Functional analysis [Rudin 1973]; and it was his book Function theory in the unit ball of $\mathbb{C}^{n}$ [Rudin 1980] that first interested me in uniform algebras.

\section{The theorem and its proof}

Theorem 2.1. On every compact $C^{\infty}$-manifold $M$ of dimension greater than or equal to 3 , there exists a nonlocal uniform algebra with maximal ideal space $M$ generated by $C^{\infty}$-smooth functions.

Before proving the theorem, we establish some technical lemmas that are used to prove the smoothness assertion. Throughout the paper $D$ will denote the open unit disc $\{z \in \mathbb{C}:|z|<1\}$ and $L$ will denote the open annulus $\{z \in \mathbb{C}: 3<|z|<4\}$.

Lemma 2.2. There exists a $C^{\infty}$-smooth map $\Phi: \mathbb{R}^{2} \rightarrow \mathbb{R}^{2}$ that takes the closed unit disc $\bar{D}$ one-to-one onto itself and satisfies $\Phi(x, 0)=(1,0)$ for all $x \geq 1$.

Proof. Let $U=\left\{(x, y) \in \mathbb{R}^{2}: \frac{1}{2}<x\right.$ and $\left.-\frac{1}{2}<y<\frac{1}{2}\right\}$, and define $\varphi: U \rightarrow \mathbb{R}^{2}$ by $\varphi(x, y)=\left(x-1+\sqrt{1-y^{2}}, y\right)$. Then $\varphi$ is a diffeomorphism of $U$ onto the neighborhood $\varphi(U)$ of the point $\varphi(1,0)=(1,0)$.

Let $\alpha: \mathbb{R} \rightarrow \mathbb{R}$ be a $C^{\infty}$-function that is strictly increasing on the interval $(-\infty, 1]$ and that satisfies

$$
\alpha(x)= \begin{cases}x & \text { for } x \leq \frac{3}{4} \\ 1 & \text { for } x \geq 1\end{cases}
$$


Let $\beta: \mathbb{R} \rightarrow[0,1]$ be a $C^{\infty}$-function such that

$$
\beta(y)= \begin{cases}0 & \text { for } y=0 \\ 1 & \text { for }|y| \geq \frac{1}{4}\end{cases}
$$

Define $\sigma: U \rightarrow \mathbb{R}$ by $\sigma(x, y)=(1-\beta(y)) \alpha(x)+\beta(y) x$. Then the reader can verify that the map $\Psi: U \rightarrow U$ given by

$$
\Psi(x, y)=(\sigma(x, y), y)
$$

is well-defined (that is, it takes $U$ into $U$ ) and satisfies

(1) $\Psi(x, 0)=(1,0)$ for all $x \geq 1$,

(2) $\Psi\left(\frac{1}{2}, y\right)=\left(\frac{1}{2}, y\right)$ for all $y$,

(3) $\Psi(1, y)=(1, y)$ for all $y$, and

(4) $\Psi(x, y)=(x, y)$ whenever either $x \leq \frac{3}{4}$ or $|y| \geq \frac{1}{4}$.

Regarded as a function of the first variable, $\sigma$ is strictly increasing on the interval $\left[\frac{1}{2}, 1\right]$ for each fixed $y$, so (2) and (3) imply that $\Psi$ maps the square $\left[\frac{1}{2}, 1\right] \times\left[-\frac{1}{2}, \frac{1}{2}\right]$ one-to-one onto itself.

Define $\Phi: \varphi(U) \rightarrow \mathbb{R}^{2}$ by $\Phi=\varphi \circ \Psi \circ \varphi^{-1}$. Then by (1), $\Phi$ takes the constant value $(1,0)$ on $\{(x, y): x \geq 1, y=0\}$. Also, $\Phi$ maps $\varphi(U) \cap \bar{D}=\varphi\left(\left[\frac{1}{2}, 1\right] \times\left[-\frac{1}{2}, \frac{1}{2}\right]\right)$ one-to-one onto itself. Of course $\Phi$ is of class $C^{\infty}$. By (4), outside of the closed subset $\varphi\left(\left[\frac{3}{4}, \infty\right) \times\left[-\frac{1}{4}, \frac{1}{4}\right]\right)$ of $\varphi(U)$, the map $\Phi$ is the identity. Hence, $\Phi$ can be extended to a $C^{\infty}$-map on all of $\mathbb{R}^{2}$ by making $\Phi$ the identity outside of $\varphi(U)$. Then $\Phi$ takes $\bar{D}$ one-to-one onto itself and satisfies $\Phi(x, 0)=(1,0)$ for all $x \geq 1$.

Lemma 2.3. There exists a $C^{\infty}$-smooth map $F: \mathbb{R}^{2} \rightarrow \mathbb{R}^{2}$ that takes the closed disc $\left\{(x, y):(x-2)^{2}+y^{2} \leq 1\right\}$ homeomorphically onto $\bar{D}$, takes the closed disc $\left\{(x, y):(x-5)^{2}+y^{2} \leq 1\right\}$ homeomorphically onto $\bar{D}$ as well, and takes the constant value $(1,0)$ on the set $\{(x, y): 3 \leq x \leq 4, y=0\}$.

Proof. Let $\Phi$ be the map in Lemma 2.2. Define $F_{1}, F_{2}: \mathbb{R}^{2} \rightarrow \mathbb{R}^{2}$ by $F_{1}(x, y)=$ $\Phi(x-2, y)$ and $F_{2}(x, y)=\Phi(5-x, y)$. Let $\left\{\varphi_{1}, \varphi_{2}\right\}$ be a $C^{\infty}$-partition of unity on $\mathbb{R}^{2}$ subordinate to the cover consisting of $\left\{(x, y): x<3+\frac{2}{3}\right\}$ and $\left\{(x, y): x>3+\frac{1}{3}\right\}$, and define $F: \mathbb{R}^{2} \rightarrow \mathbb{R}^{2}$ by $F=\varphi_{1} F_{1}+\varphi_{2} F_{2}$. Then $F$ has all the required properties.

Proof of Theorem 2.1. We divide the proof into steps.

Step 1. We define a certain uniform algebra $U$ from which the desired uniform algebra will be obtained, and we determine the maximal ideal space $\mathfrak{M}_{\bullet}$ and Gelfand transform for $U$.

Let $A(D)$ denote the disc algebra on the disc (the algebra of continuous functions on $\bar{D}$ that are holomorphic on $D$ ), and let $R_{b}(L)$ denote the annulus algebra on $\partial L$ 
(the algebra of continuous functions $\partial L$ that have holomorphic extension to the annulus $L$ ). Let $S$ be the singular inner function given by

$$
S(z)=\exp \left(\frac{z+1}{z-1}\right)
$$

and let

$$
I=\{S g: g \in A(D) \text { and } g(1)=0\} .
$$

Then $I$ is a proper closed ideal in $A(D)$. (See [Hoffman 1962, pp. 83-84].)

For uniform algebras $F$ and $G$ on spaces $X$ and $Y$ respectively, we take the tensor product $F \otimes G$ to be the linear span of the functions of the form

$$
(f \otimes g)(x, y)=f(x) g(y),
$$

with $f \in F$ and $g \in G$. Now let $U$ be the uniform algebra on $\partial L \times \bar{D}$ generated by $C(\partial L) \otimes I$ and $R_{b}(L) \otimes A(D)$, or, equivalently, set

$$
U=\overline{(C(\partial L) \otimes I)+\left(R_{b}(L) \otimes A(D)\right)} .
$$

We now apply the material from [Sidney 1968, p. 135] with $A^{\prime}=C(\partial L), B=A(D)$, $X=\mathfrak{M}_{A^{\prime}}=\partial L, Y=\mathfrak{M}_{B}=\bar{D}$, and $A=R_{b}(L)$. As in that reference, let $\tau: A \rightarrow \mathcal{U}$ and $\eta: B \rightarrow \mathcal{U}$ be the isometric isomorphisms $\tau(a)=a \otimes 1$ and $\eta(b)=1 \otimes b$, and define $\pi: \mathfrak{M}_{\iota_{u}} \rightarrow \mathfrak{M}_{A} \times \mathfrak{M}_{B}$ by $\pi(\varphi)=\left(\tau_{*}(\varphi), \eta_{*}(\varphi)\right)$, where $\tau_{*}: \mathfrak{M}_{{ }_{u}} \rightarrow \mathfrak{M}_{A}$ and $\eta_{*}: \mathfrak{M}_{{ }_{U}} \rightarrow \mathfrak{M}_{B}$ are the dual maps. Then by [Sidney 1968, Theorem 3.3], $\pi$ maps

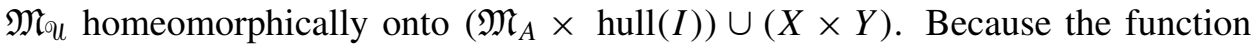
$(z-1) S$ is in $I$ and vanishes at no point of $\bar{D}$ other than 1 , the hull of $I$ is $\{1\}$. Thus, since $\mathfrak{M}_{A}=\bar{L}$, we get that the maximal ideal space of $U$ can be identified with

$$
(\bar{L} \times\{1\}) \cup((\partial L) \times \bar{D}) \subset \mathbb{C}^{2} .
$$

Under the identification, each point of $(\partial L) \times \bar{D}$ is identified with the corresponding point evaluation functional, so the Gelfand transform of a function $f \in \mathcal{U}$ gives an extension of $f$ to the annulus $L \times\{1\}$. The reader can check that this extension is the holomorphic extension of the annulus algebra function $x \mapsto f(x, 1)$ to $L$.

Step 2. We show that $U$ is nonlocal by showing that the function

$$
h \in C((\bar{L} \times\{1\}) \cup((\partial L) \times \bar{D})),
$$

given by

$$
h(w, z)= \begin{cases}0 & \text { for }(w, z) \in(\bar{L} \times\{1\}) \cup(\{|w|=4\} \times \bar{D}), \\ z-1 & \text { for }(w, z) \in(\bar{L} \times\{1\}) \cup(\{|w|=3\} \times \bar{D}),\end{cases}
$$

is locally in $U$ but not in $U$.

That $h$ is locally in $U$ is clear because the interiors (relative to $\mathfrak{M}_{\boldsymbol{U}}$ ) of the sets $(\bar{L} \times\{1\}) \cup(\{|w|=4\} \times \bar{D})$ and $(\bar{L} \times\{1\}) \cup(\{|w|=3\} \times \bar{D})$ cover $\mathfrak{M}_{\iota}$ and the 
functions 0 and $z-1$ each lie in $\mathcal{~}$. To show that $h$ is not in $U$, we exhibit a measure on $(\partial L) \times \bar{D}$ that annihilates $\mathcal{U}$ but does not annihilate $h$. Because the function that is 1 on the circle $\{|w|=3\}$ and 0 on the circle $\{|w|=4\}$ is not in $R_{b}(L)$, there is a measure $\mu$ on $\partial L$ that annihilates $R_{b}(L)$ but does not annihilate this function. Also, because the function $z-1$ is not in the ideal $I$, there is a measure $v$ on $\bar{D}$ that annihilates $I$ but such that $\int_{\bar{D}}(z-1) d v(z)=1$. Now for $f \in C(\partial L)$ and $g \in I$,

$$
\int_{(\partial L) \times \bar{D}} f(w) g(z) d(\mu \times v)(w, z)=\int_{\partial R} f d \mu \cdot \int_{\bar{D}} g d v=0,
$$

and the same equation holds also for $f \in R_{b}(L)$ and $g \in A(D)$. Consequently, $\mu \times v$ annihilates $\overline{(C(\partial L) \otimes I)+\left(R_{b}(L) \otimes A(D)\right)}=U$. However,

$$
\begin{aligned}
\int_{(\partial L) \times \bar{D}} & h(w, z) d(\mu \times v)(w, z) \\
& =\int_{\{|w|=3\} \times \bar{D}} h(w, z) d(\mu \times v)(w, z)+\int_{\{|w|=4\} \times \bar{D}} h(w, z) d(\mu \times v)(w, z) \\
& =\int_{\{|w|=3\}} \int_{\bar{D}}(z-1) d v(z) d \mu(w)+\int_{\{|w|=4\}} \int_{\bar{D}} 0 d v(z) d \mu(w) \\
& =\int_{\{|w|=3\}} 1 d \mu+\int_{\{|w|=4\}} 0 d \mu \neq 0 .
\end{aligned}
$$

Thus $h$ is not in $U$.

Step 3. We show that there is a dense set of functions in $I$ that extend to $C^{\infty}$ functions on $\mathbb{C}$.

Let $A^{\infty}(D)$ denote the algebra of functions in $A(D)$ whose complex derivatives to all orders also lie in $A(D)$. The boundary function of each function in $A^{\infty}(D)$ belongs to $C^{\infty}(\partial D)$, and $A^{\infty}(D)$ is a topological algebra with the topology induced by $C^{\infty}(\partial D)$. Each function in $A^{\infty}(D)$ extends to a $C^{\infty}$-function on $\mathbb{C}$. (This follows from Whitney's extension theorem [Boggess 1991, Theorem 2 in Section 5.3].)

Let $J=\left\{S g: g \in A^{\infty}(D)\right.$ and $\left.S g \in A^{\infty}(D)\right\}$. Note that $J$ is an ideal in $A^{\infty}(D)$. Consider the closure $\bar{J}$ of $J$ in the disc algebra $A(D)$. One easily checks that $\bar{J}$ is a (closed) ideal in $A(D)$. By [Taylor and Williams 1970, Theorem 3.3], there is an outer function $h$ in $A^{\infty}(D)$ with $h^{(n)}(1)=0$ for all $n=0,1,2, \ldots$ (that is, vanishing to infinite order at 1) with no other zeros on $\bar{D}$. Then by [Taylor and Williams 1970, Theorem 4.7], the function $S h$ is in $J$. Because $S h$ vanishes only at $z=1$, we conclude from the Beurling-Rudin theorem [Rudin 1957] (or see [Hoffman 1962, pp. 82-89] that

$$
\bar{J}=\left\{g \exp \left(r \frac{z+1}{z-1}\right): g \in A(D) \text { and } g(1)=0\right\}
$$


for some $r \geq 0$. Obviously

$$
\bar{J} \subset\left\{g \exp \left(\frac{z+1}{z-1}\right): g \in A(D) \text { and } g(1)=0\right\},
$$

so $r \geq 1$. Because $h$ is outer, $S h$ is not of the form $g \exp [r(z+1) /(z-1)]$ for any $r>1$. Thus $r=1$, that is, $\bar{J}=I$. Because the functions in $J$ extend to $C^{\infty}$-functions on $\mathbb{C}$, this completes Step 3 .

Step 4. We show that there is a dense set of functions in $\mathcal{U}$, regarded as a uniform algebra on $\mathfrak{M}_{u}=(\bar{L} \times\{1\}) \cup((\partial L) \times \bar{D})$, that extend to $C^{\infty}$-functions on $\mathbb{C}^{2}$.

Recall that $U$, as a uniform algebra on $(\partial L) \times \bar{D}$, is the closed linear span of the functions of the form $(f \otimes g)(x, y)=f(x) g(y)$, where either $f \in R_{b}(L)$ and $g \in A(D)$, or else $f \in C(\partial L)$ and $g \in I$. Thus, it suffices to show that each of these functions $f \otimes g$ can be uniformly approximated on $(\partial L) \times \bar{D}$ by functions $C^{\infty}$ on $\mathbb{C}^{2}$ that lie in $\mathcal{U}$ on $\mathfrak{M}_{\iota_{u}}$. For $f \in R_{b}(L)$ and $g \in A(D)$, we trivially obtain sequences $\left(f_{n}\right)$ and $\left(g_{n}\right)$ of functions $C^{\infty}$ on $\mathbb{C}$ with $f_{n} \mid \bar{L} \in R(\bar{L})$ and $g_{n} \mid \bar{D} \in A(D)$ such that $f_{n} \rightarrow f$ and $g_{n} \rightarrow g$ uniformly on $\partial L$ and $\bar{D}$ respectively. Then $f_{n} \otimes g_{n}$ gives the required approximation of $f \otimes g$. For $f \in C(\partial L)$, there is a sequence $\left(f_{n}\right)$ of functions $C^{\infty}$ on $\mathbb{C}$ with $f_{n} \rightarrow f$ uniformly on $\partial L$, and for $g \in I$, there is, by Step 3, a sequence $\left(g_{n}\right)$ of functions $C^{\infty}$ on $\mathbb{C}$ with $g_{n} \mid \bar{D} \in I$ and $g_{n} \rightarrow g$ uniformly on $\bar{D}$. Then $f_{n} \otimes g_{n}$ is of course $C^{\infty}$ on $\mathbb{C}$ and in $U$ on $(\partial L) \times \bar{D}$, and $f_{n} \otimes g_{n} \rightarrow f \otimes g$ uniformly there. Also, because $g_{n}(1)=0$, we have $f_{n} \otimes g_{n}=0$

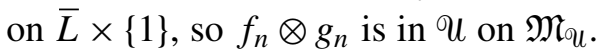

Step 5. We show that there is a $C^{\infty}$-map $G: \mathbb{R}^{3} \backslash\left\{x_{1}=x_{2}=0\right\} \rightarrow \mathbb{C}^{2}$ that takes

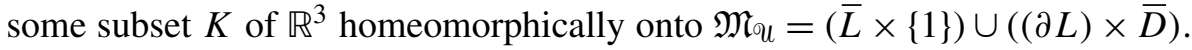

Let $K=K_{L} \cup K_{1} \cup K_{2}$, where

$$
\begin{aligned}
& K_{L}=\left\{\left(x_{1}, x_{2}, x_{3}\right) \in \mathbb{R}^{3}: 3 \leq \sqrt{x_{1}^{2}+x_{2}^{2}} \leq 4, x_{3}=0\right\}, \\
& K_{1}=\left\{\left(x_{1}, x_{2}, x_{3}\right) \in \mathbb{R}^{3}:\left(\sqrt{x_{1}^{2}+x_{2}^{2}}-2\right)^{2}+x_{3}^{2} \leq 1\right\}, \text { and } \\
& K_{2}=\left\{\left(x_{1}, x_{2}, x_{3}\right) \in \mathbb{R}^{3}:\left(\sqrt{x_{1}^{2}+x_{2}^{2}}-5\right)^{2}+x_{3}^{2} \leq 1\right\} .
\end{aligned}
$$

Let $\rho: \mathbb{R} \rightarrow \mathbb{R}$ be a $C^{\infty}$-function such that $\rho([1,3])=\{3\}$, on the interval $[3,4]$ the function $\rho$ strictly increases from 3 to 4 , and $\rho([4,6])=\{4\}$. Let $F$ be the map in Lemma 2.3 and define $G: \mathbb{R}^{3} \backslash\left\{x_{1}=x_{2}=0\right\} \rightarrow \mathbb{C}^{2}$ by

$$
G\left(x_{1}, x_{2}, x_{3}\right)=\left(\rho\left(\sqrt{x_{1}^{2}+x_{2}^{2}}\right) \frac{\left(x_{1}+x_{2} i\right)}{\sqrt{x_{1}^{2}+x_{2}^{2}}}, F\left(\sqrt{x_{1}^{2}+x_{2}^{2}}, x_{3}\right)\right) .
$$

Clearly, $G$ is of class $C^{\infty}$. One checks easily that $G$ takes $K_{L}$ one-to-one onto $\bar{L} \times\{1\}$, takes $K_{1}$ one-to-one onto $\{|z|=3\} \times \bar{D}$, and takes $K_{2}$ one-to-one onto $\{|z|=4\} \times \bar{D}$. Thus, $G$ takes $K$ onto $\mathfrak{M}_{\text {ฯ }}$, and a little more thought shows that $G$ 
is one-to-one on $K$. Since $K$ is compact, we conclude that $G$ takes $K$ homeomorphically onto $\mathfrak{M}_{\imath}$.

Step 6. We complete the proof of the theorem.

The uniform algebra $B=\{f \circ G: f \in \mathcal{U}\}$ on $K$ is clearly isomorphic to $U$. By Step 2, $B$ is nonlocal, and by Step 4, $B$ is generated by functions that extend to be $C^{\infty}$ on $\mathbb{R}^{3} \backslash\left\{x_{1}=x_{2}=0\right\}$.

Given an arbitrary compact $C^{\infty}$-manifold $M$ of dimension greater than or equal to 3 , choose an embedding of a neighborhood of $K$ into $M$, identify $K$ with its image in $M$, and define a uniform algebra $A$ on $M$ by taking all continuous functions on $M$ whose restrictions to $K$ lie in $B$. Then the maximal ideal space of $A$ is $M$ by [Bear 1959, Theorem 4], $A$ is nonlocal by [Izzo 2010, Lemma 2.5], and $A$ is generated by $C^{\infty}$-smooth functions by [Izzo 2009, Lemma 2.1].

\section{Acknowledgement}

I would like to thank Stuart Sidney for explaining to me his approach to nonlocal uniform algebras, without which I would not have been able to construct the examples given here.

\section{References}

[Bear 1959] H. S. Bear, “Complex function algebras”, Trans. Amer. Math. Soc. 90 (1959), 383-393. MR 21 \#5889 Zbl 0086.31602

[Boggess 1991] A. Boggess, CR manifolds and the tangential Cauchy-Riemann complex, CRC Press, Boca Raton, FL, 1991. MR 94e:32035 Zbl 0760.32001

[Gamelin 1984] T. W. Gamelin, Uniform algebras, 2nd ed., Chelsea, New York, 1984. MR 53 \#14137 Zbl 0213.40401

[Hoffman 1962] K. Hoffman, Banach spaces of analytic functions, Prentice-Hall, Englewood Cliffs, NJ, 1962. MR 24 \#A2844 Zbl 0117.34001

[Izzo 2009] A. J. Izzo, "Uniform algebras on the sphere invariant under group actions", Math. Ann. 344:4 (2009), 989-995. MR 2010f:46079 Zbl 1184.46049

[Izzo 2010] A. J. Izzo, "Localization for uniform algebras generated by smooth functions on twomanifolds”, Bull. Lond. Math. Soc. 42:4 (2010), 652-660. MR 2011g:46090 Zbl 1203.46033

[Kallin 1963] E. Kallin, "A nonlocal function algebra", Proc. Nat. Acad. Sci. USA 49 (1963), 821824. MR 27 \#2878 Zbl 0142.10103

[Rudin 1957] W. Rudin, "The closed ideals in an algebra of analytic functions", Canad. J. Math. 9 (1957), 426-434. MR 19,641c Zbl 0080.31703

[Rudin 1973] W. Rudin, Functional analysis, McGraw-Hill, New York, 1973. MR 51 \#1315 Zbl 0253.46001

[Rudin 1974] W. Rudin, Real and complex analysis, 2nd ed., McGraw-Hill, New York, 1974. MR 49 \#8783 Zbl 0278.26001

[Rudin 1976] W. Rudin, Principles of mathematical analysis, 3rd ed., McGraw-Hill, New York, 1976. MR 52 \#5893 Zbl 0346.26002 
[Rudin 1980] W. Rudin, Function theory in the unit ball of $\mathbb{C}^{n}$, Grundlehren der Mathematischen Wissenschaften 241, Springer, New York, 1980. MR 82i:32002 Zbl 0495.32001

[Sidney 1968] S. J. Sidney, "Properties of the sequence of closed powers of a maximal ideal in a sup-norm algebra", Trans. Amer. Math. Soc. 131 (1968), 128-148. MR 36 \#5701 Zbl 0172.18101

[Taylor and Williams 1970] B. A. Taylor and D. L. Williams, "Ideals in rings of analytic functions with smooth boundary values”, Canad. J. Math. 22 (1970), 1266-1283. MR 42 \#7905 Zbl 0204.44302

Received August 7, 2011. Revised November 29, 2011.

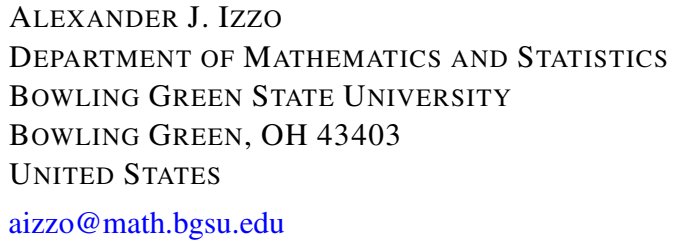




\title{
PACIFIC JOURNAL OF MATHEMATICS
}

\author{
http://pacificmath.org \\ Founded in 1951 by \\ E. F. Beckenbach (1906-1982) and F. Wolf (1904-1989)
}

\section{EDITORS}

V. S. Varadarajan (Managing Editor)

Department of Mathematics

University of California

Los Angeles, CA 90095-1555

pacific@math.ucla.edu

Vyjayanthi Chari

Department of Mathematics

University of California

Riverside, CA 92521-0135

chari@math.ucr.edu

\section{Robert Finn}

Department of Mathematics Stanford University

Stanford, CA 94305-2125

finn@math.stanford.edu

Kefeng Liu

Department of Mathematics

University of California

Los Angeles, CA 90095-1555

liu@math.ucla.edu
Darren Long

Department of Mathematics

University of California

Santa Barbara, CA 93106-3080

long@math.ucsb.edu

Jiang-Hua Lu

Department of Mathematics

The University of Hong Kong

Pokfulam Rd., Hong Kong jhlu@maths.hku.hk

Alexander Merkurjev

Department of Mathematics

University of California

Los Angeles, CA 90095-1555

merkurev@math.ucla.edu
Sorin Popa

Department of Mathematics University of California

Los Angeles, CA 90095-1555 popa@math.ucla.edu

Jie Qing

Department of Mathematics

University of California

Santa Cruz, CA 95064

qing@cats.ucsc.edu

Jonathan Rogawski

Department of Mathematics

University of California

Los Angeles, CA 90095-1555

jonr@math.ucla.edu

\section{PRODUCTION}

pacific@math.berkeley.edu

\section{SUPPORTING INSTITUTIONS}

ACADEMIA SINICA, TAIPEI

CALIFORNIA INST. OF TECHNOLOGY INST. DE MATEMÁTICA PURA E APLICADA KEIO UNIVERSITY

MATH. SCIENCES RESEARCH INSTITUTE NEW MEXICO STATE UNIV.

OREGON STATE UNIV.

\author{
STANFORD UNIVERSITY \\ UNIV. OF BRITISH COLUMBIA \\ UNIV. OF CALIFORNIA, BERKELEY \\ UNIV. OF CALIFORNIA, DAVIS \\ UNIV. OF CALIFORNIA, LOS ANGELES \\ UNIV. OF CALIFORNIA, RIVERSIDE \\ UNIV. OF CALIFORNIA, SAN DIEGO \\ UNIV. OF CALIF., SANTA BARBARA
}

\author{
UNIV. OF CALIF., SANTA CRUZ \\ UNIV. OF MONTANA \\ UNIV. OF OREGON \\ UNIV. OF SOUTHERN CALIFORNIA \\ UNIV. OF UTAH \\ UNIV. OF WASHINGTON \\ WASHINGTON STATE UNIVERSITY
}

These supporting institutions contribute to the cost of publication of this Journal, but they are not owners or publishers and have no responsibility for its contents or policies.

See inside back cover or pacificmath.org for submission instructions.

The subscription price for 2012 is US \$420/year for the electronic version, and \$485/year for print and electronic.

Subscriptions, requests for back issues from the last three years and changes of subscribers address should be sent to Pacific Journal of Mathematics, P.O. Box 4163, Berkeley, CA 94704-0163, U.S.A. Prior back issues are obtainable from Periodicals Service Company, 11 Main Street, Germantown, NY 12526-5635. The Pacific Journal of Mathematics is indexed by Mathematical Reviews, Zentralblatt MATH, PASCAL CNRS Index, Referativnyi Zhurnal, Current Mathematical Publications and the Science Citation Index.

The Pacific Journal of Mathematics (ISSN 0030-8730) at the University of California, c/o Department of Mathematics, 969 Evans Hall, Berkeley, CA 94720-3840, is published monthly except July and August. Periodical rate postage paid at Berkeley, CA 94704, and additional mailing offices. POSTMASTER: send address changes to Pacific Journal of Mathematics, P.O. Box 4163, Berkeley, CA 94704-0163.

PJM peer review and production are managed by EditFLOW ${ }^{\mathrm{TM}}$ from Mathematical Sciences Publishers.

PUBLISHED BY PACIFIC JOURNAL OF MATHEMATICS

at the University of California, Berkeley 94720-3840

A NON-PROFIT CORPORATION

Typeset in LATEX

Copyright $(02012$ by Pacific Journal of Mathematics 


\title{
PACIFIC JOURNAL OF MATHEMATICS
}

\author{
Volume $259 \quad$ No. $1 \quad$ September 2012
}

Extension Theorems for external cusps with minimal regularity

GABRIEL ACOSTA and IGNACIO OJEA

Convergence of axially symmetric volume-preserving mean curvature flow

MARIA ATHANASSENAS and SEVVANDi KANDANAARACHCHI

On the horoboundary and the geometry of rays of negatively curved manifolds

FranÇOISE DAL'bo, Marc PEIGNÉ and ANDREA

SAMBUSETTI

Two infinite versions of the nonlinear Dvoretzky theorem

KEI FUNANO

Nonlocal uniform algebras on three-manifolds

ALEXANDER J. IZZO

Mahlo cardinals and the torsion product of primary abelian groups

PATRICK W. KEEF

Geometry of trinomials

AARON MELMAN

Drinfeld orbifold algebras

ANNE V. SHEPLER and SARAH Witherspoon

Semi-topological cycle theory I

JYH-HAUR TEH

New construction of fundamental domains for certain Mostow groups 209

TiEHONG ZHAO 